\title{
Chorda Tympani Nerve Terminal Field Maturation and Maintenance Is Severely Altered Following Changes to Gustatory Nerve Input to the Nucleus of the Solitary Tract
}

\author{
Sara L. Corson ${ }^{1}$ and David L. Hill ${ }^{2}$ \\ ${ }^{1}$ Neuroscience Graduate Program and ${ }^{2}$ Department of Psychology, University of Virginia, Charlottesville, Virginia 22904-4400
}

\begin{abstract}
Neural competition among multiple inputs can affect the refinement and maintenance of terminal fields in sensory systems. In the rat gustatory system, the chorda tympani, greater superficial petrosal, and glossopharyngeal nerves have distinct but overlapping terminal fields in the first central relay, the nucleus of the solitary tract. This overlap is largest at early postnatal ages followed by a significant refinement and pruning of the fields over a 3 week period, suggesting that competitive mechanisms underlie the pruning. Here, we manipulated the putative competitive interactions among the three nerves by sectioning the greater superficial petrosal and glossopharyngeal nerves at postnatal day 15 (P15), P25, or at adulthood, while leaving the chorda tympani nerve intact. The terminal field of the chorda tympani nerve was assessed $35 \mathrm{~d}$ following nerve sections, a period before the sectioned nerves functionally regenerated. Regardless of the age when the nerves were cut, the chorda tympani nerve terminal field expanded to a volume four times larger than sham controls. Terminal field density measurements revealed that the expanded terminal field was similar to $\mathrm{P} 15$ control rats. Thus, it appears that the chorda tympani nerve terminal field defaults to its early postnatal field size and shape when the nerves with overlapping fields are cut, and this anatomical plasticity is retained into adulthood. These findings not only demonstrate the dramatic and lifelong plasticity in the central gustatory system, but also suggest that corresponding changes in functional and taste-related behaviors will accompany injury-induced changes in brainstem circuits.
\end{abstract}

\section{Introduction}

Age-related refinement of afferent terminal fields is a common characteristic of many sensory systems. This refinement often occurs in central structures where multiple inputs converge onto the same target area, suggesting that interactions among terminal fields shape their maturation. Examples are seen in the developing visual, somatosensory, and auditory systems (Pallas and Hoy, 1986; Shatz, 1990; Chiaia et al., 1992; Renehan et al., 1994; Gosse et al., 2008). Interestingly, these systems often maintain the ability to reorganize in response to experimental perturbations into adulthood (Chiaia et al., 1992, 1993; Jacquin et al., 1993; Renehan et al., 1994; Foeller and Feldman, 2004).

The gustatory system has multiple primary afferent inputs projecting to a common target area in the brainstem, and these inputs are refined throughout development (Sollars et al., 2006; Mangold and Hill, 2007; May et al., 2008). Moreover, the developing gustatory system exhibits significant anatomical and functional plasticity (Lasiter and Kachele, 1990; King and Hill, 1991; Mangold and Hill, 2007, 2008; Thomas and Hill, 2008). The mechanisms that drive these changes have not been identified.

Received Jan. 10, 2011; revised March 21, 2011; accepted April 12, 2011.

Author contributions: S.L.C. and D.L.H. designed research; S.L.C. and D.L.H. performed research;S.L.C. and D.L.H. analyzed data; S.L.C. and D.L.H. wrote the paper.

This research was supported by NIH Grants DC00407 and DC006938. We extend our appreciation to Sarah L. Deal for assistance with tissue processing and data analysis, and Dr. Peter C. Brunjes for the use of his confocal laser microscope.

Correspondence should be addressed to Dr. David L. Hill, Department of Psychology, P.0. Box 400400, University of Virginia, Charlottesville, VA 22904. E-mail: dh2t@virginia.edu.

DOI:10.1523/JNEUROSCI.0151-11.2011

Copyright $\odot 2011$ the authors $\quad 0270-6474 / 11 / 317591-13 \$ 15.00 / 0$
The chorda tympani, greater superficial petrosal, and glossopharyngeal nerves all carry orosensory information to the rostral nucleus of the solitary tract (NTS), where they form distinct but overlapping terminal fields (May and Hill, 2006). The terminal fields of the three nerves undergo morphological changes throughout postnatal development, with the chorda tympani nerve terminal field undergoing the greatest change (Sollars et al., 2006; Mangold and Hill, 2008). In particular, the volume of the rat chorda tympani nerve terminal field decreases by $57 \%$ from postnatal day 15 (P15) to P25, and then decreases another $41 \%$ by P35, at which age the volume remains constant into adulthood (Mangold and Hill, 2008). This age-related decrease in the chorda tympani nerve terminal field volume is accompanied by a decrease in the amount of shared terminal field with the glossopharyngeal nerve. The overlap of terminal field between the two nerves changes from 80 to $20 \%$ between P15 and P25. The amount of overlap between the chorda tympani and greater superficial petrosal nerves $(\sim 80 \%)$ does not change with age. To test the hypothesis that the developmental decrease in the chorda tympani nerve terminal field is influenced by interactions with the other two nerves, we unilaterally sectioned the greater superficial petrosal and glossopharyngeal nerves at P15, P25, or adulthood and left the chorda tympani nerve intact. The terminal field morphology of the chorda tympani nerve was then examined before functional regeneration occurred (i.e., $35 \mathrm{~d}$ after nerve section) in each of the three groups. The results show that the chorda tympani nerve terminal field volume remains at, or expands to, the immature volume seen in intact P15 rats, regardless of the age at which the other two nerves were sectioned. This indi- 
cates that significant interactions among the three terminal fields shape terminal field development and a remarkable degree of plasticity in the gustatory brainstem is maintained throughout life.

\section{Materials and Methods}

\section{Animals}

All experiments were approved by the University of Virginia Animal Care and Use Committee and followed guidelines set forth by the National Institutes of Health and the Society for Neuroscience. Pregnant dams and P65 (adult) female rats were purchased from Harlan. The male and female offspring of the pregnant dams born in the vivarium at the University of Virginia were used in P15 and P25 experiments. Animals were considered to be adults at P65, when terminal field volume and nerve taste-response profiles are stable and mature (Hill and Almli, 1980; Ferrell et al., 1981; Mangold and Hill, 2008).

\section{Nerve cuts}

Figure 1 shows the circuitry of the peripheral gustatory system with the three nerves of interest and their respective receptive fields and terminal fields. At P15 $(n=6), \mathrm{P} 25(n=5)$, or adulthood $(n=5)$, the right greater superficial petrosal and glossopharyngeal nerves were sectioned distal to their respective ganglion (Fig. $1)$. Rats were sedated with an intramuscular injection of $0.32 \mathrm{mg} / \mathrm{kg}$ Domitor (medetomidine hydrochloride; Pfizer Animal Health) and anesthetized intramuscularly with $40 \mathrm{mg} / \mathrm{kg}$ Ketaset (ketamine hydrochloride; Fort Dodge Animal Health). Body temperature was maintained with a water-circulating heating pad. After positioning in a nontraumatic head holder, a ventral approach was taken to expose the greater superficial petrosal nerve. The greater superficial petrosal nerve was sectioned near the geniculate ganglion in the tympanic bulla. The glossopharyngeal nerve was approached medially to the tympanic bulla and sectioned (May and Hill, 2006; Mangold and Hill, 2008). After both nerves were cut, the incision was closed with sutures and an intramuscular injection of $1.6 \mathrm{mg} / \mathrm{kg}$ Antisedan (atipamezole hydrocholoride; Pfizer Animal Health) was given to reverse anesthesia. For sham animals, the same surgical approach was taken with the exception that the nerves were not sectioned (P15, $n=4$; adult, $n=5)$.

For animals used in neurophysiological recordings from the glossopharyngeal nerve, only the left glossopharyngeal nerve was sectioned using the same surgical approach as described above.

\section{Fluorescent anterograde nerve labeling}

Thirty-five days following nerve transection, the chorda tympani nerve ipsilateral to the nerve cuts was labeled with biotinylated dextran amine (BDA; Invitrogen). We showed earlier that all three terminal fields were mature by P50 (P15 + 35 d survival) (Mangold and Hill, 2007). Therefore, all of our measurements here were done at an age when the chorda tympani nerve terminal field was mature in controls. The chorda tympani nerve was accessed following the same procedure described for the greater superficial petrosal nerve section. The chorda tympani nerve was sectioned, crystals of BDA were applied to the proximal cut end of the nerve, and the incision was closed with sutures. After a $48 \mathrm{~h}$ survival period, animals were deeply anesthetized with an intraperitoneal injection of $2.4 \mathrm{mg} / \mathrm{kg}$ urethane (ethyl-carbamate; Sigma-Aldrich) and perfused transcardially with Krebs-Henseleit buffer, $\mathrm{pH} 7.3$, followed by $8 \%$ paraformaldehyde, $\mathrm{pH}$ 7.2.

Previous work showed that anterograde tracers placed on the chorda tympani nerve do not inadvertently label other nerves. Moreover, the tracer labels the full complement of chorda tympani nerve fibers (Mangold and Hill, 2008).

\section{Tissue preparation}

Brains were removed and postfixed overnight in $8 \%$ paraformaldehyde. The medulla was blocked, and a vibratome was used to section the tissue horizontally in $50 \mu \mathrm{m}$ sections. This allowed for visualization of the entire rostral-caudal extent of the terminal field in the NTS for each section (see below for details). Sections were incubated in PBS, pH 7.2, containing $0.2 \%$ Triton and 1:500 streptavidin Alexa Fluor 488 (Invitrogen) forl h.

\section{Confocal microscopy and analyses of terminal fields}

Imaging. Chorda tympani nerve terminal fields were imaged using an Olympus IX70 microscope fitted with a Fluoview v5.0 scanning system (Olympus America) and a $10 \times$ objective [UPlanSApo; numerical aperture $(\mathrm{NA})=0.40]$. Sequential optical sections were captured every $3 \mu \mathrm{m}$ for each $50 \mu \mathrm{m}$ section. Images were obtained with settings adjusted so that pixel intensities were near (but not at) saturation. A transmitted light image at $4 \times($ UPlanSApo; $\mathrm{NA}=0.13)$ and at $10 \times$ was captured for every physical section containing the labeled terminal field. This permitted an accurate registration of common dorsal to ventral brainstem sections among animals using common brainstem landmarks $(4 \times)$ and identification of NTS borders $(10 \times)$.

Analyses of total terminal field volume. Quantification of terminal field volume was achieved through the use of ImageJ software. To aid in comparisons among sections, each image stack was initially rotated in ImageJ so that the solitary tract was oriented vertically (Fig. 2). With the corresponding transmitted light image used as a reference, the border of the NTS was outlined for each physical section. The stack was then cropped to include only the NTS (Fig. 2). The maximum entropy thresholder algorithm (Sahoo et al., 1988) was then applied to the pixel intensity frequency histogram for the entire image stack (i.e., all tiff files of every optical image). This yielded an 8 bit, binary image stack of the pixels above threshold representing labeled terminal field. Figure $2 \mathrm{~A}$ shows the raw image for an optical section in grayscale overlaid by the thresholded image in red. Figure $2 B$ shows the binary image of the pixels above threshold in the optical section and represents the data that were analyzed. A particle analysis was performed to quantify the pixel area above threshold. That is, the number of pixels above threshold for each optical section were summed by ImageJ, converted into area by multiplying the 


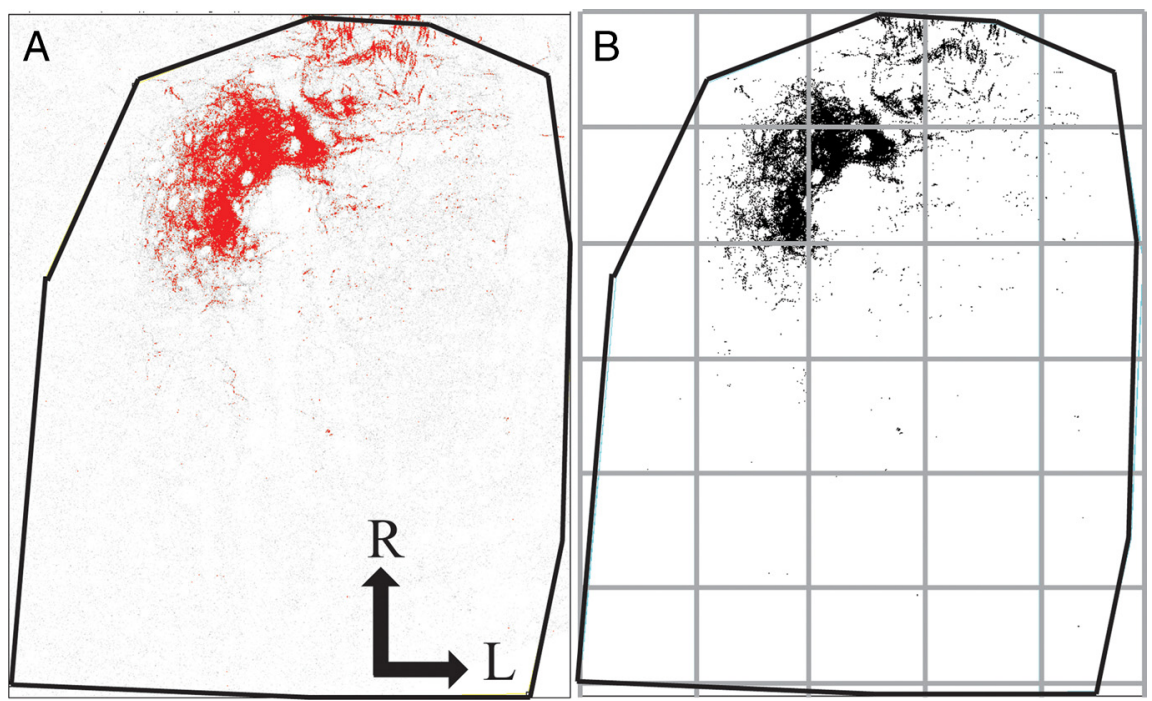

Figure 2. Method for measuring terminal field volume and density. Confocal stacks of the chorda tympani nerve terminal field were rotated in ImageJ so the solitary tract was orientated vertically, the borders of the NTS were defined, and the stack was cropped to include only the NTS. The maximum entropy thresholder algorithm was applied to the stack. $\boldsymbol{A}$, For each optical section, pixels above threshold (red) were overlaid on the raw image (gray). $\boldsymbol{B}$, A binary image of the pixels above threshold represents data that were analyzed using particle analysis. The NTS in the horizontal plane (outline shown in black lines) was subdivided into $200 \times 200$ pixel uniform grid boxes for density analysis, with the grid (see gray boxes) arranged relative to the NTS with the most medial and rostral borders of the NTS as the 0,0 coordinate. R, Rostral; L, lateral.

number of pixels by pixel size $(1.38 \times 1.38 \mu \mathrm{m})$, and then multiplied by $3 \mu \mathrm{m}$ (i.e., distance between optical sections) to determine the volume of BDA label in each physical section. Volumes from each physical section were summed to yield the total terminal field volume for each rat. The resultant volume represents an unbiased experimenter measure of the amount of label.

We realize that the diameter of some of the unmyelinated chorda tympani nerve axons may be smaller than the pixel size obtained at $10 \times$ (i.e., $<1.38 \mu \mathrm{m}$ ) (Whitehead and Frank, 1983). To address this, we examined the same region of interest at $10 \times$ and at $20 \times(0.69 \mu \mathrm{m}$ per pixel $)$ in a sham and in an adult-cut rat and found that the calculated volumes were within $10 \%$ of each other. Interestingly, the larger volumes in both cases were with the $20 \times$ measurement, perhaps due to the higher resolution imaging at $20 \times$. Additionally, we chose to include axons (e.g., the solitary tract) along with the terminal field for all animals in our analyses because of the difficulty in accurately cropping out these elements from each optical section. Accordingly, the absolute volumes that we show here include the composite terminal field and axons. Since there is no obvious reorganization of nerve tracts following the experimental manipulation, we make the assumption that including the solitary tract in our measurements has a similar effect among groups.

Analyses of terminal field volume and density of label in dorsal-ventral zones. To identify where the potential reorganization of the chorda tympani nerve terminal field occurred following our experimental manipulation, we subdivided the NTS into $x, y$, and $z$ planes. For the medial-lateral and rostral-caudal analyses ( $x$ and $y$ ), the NTS in the horizontal plane was subdivided into uniform grid boxes of $200 \times 200$ pixels $(276 \times 276 \mu \mathrm{m})$ (Fig. $2 B$, gray lines). The grid was arranged relative to the NTS with the most medial and most rostral borders of the NTS as the 0,0 coordinate. The density of terminal field label was calculated in each grid box for each physical section by dividing the chorda tympani terminal field volume within a grid box by the volume of the respective portion of the NTS contained within the grid box (i.e., volume of terminal field label/volume of the NTS within the grid box). Thus, for grid boxes that extended beyond the borders of the NTS (Fig. $2 B$, upper left grid box), only the volume of the NTS contained within the grid box was used for analyses. In the case of the grid box in the upper left portion of Figure $2 B$, the volume of the NTS was $22,908.82 \mu \mathrm{m}^{3}$, whereas the grid box in the second row and second column contained the maximum volume of the NTS $\left(2,646,471.96 \mu \mathrm{m}^{3}\right)$.
For analyses in the dorsal-ventral planes $(z$ plane), we examined the volume of labeled terminal field in five dorsal-ventral zones, each consisting of $100 \mu \mathrm{m}$ (i.e., 2 horizontal sections/zone) (Fig. 3). This type of analyses is similar to what has been done previously (King and Hill, 1991; Krimm and Hill, 1997; May and Hill, 2006; Sollars et al., 2006; Mangold and Hill, 2008), with the exception that the current data were analyzed for five instead of three dorsal-ventral zones. The orientation of the NTS within the brainstem is such that the caudalmost portion of the NTS is dorsal to the rostralmost portion (Mangold and Hill, 2007) (Fig. $3 F$ ). That is, the NTS extends ventrally and rostrally from the dorsal-most extent of the NTS. Therefore, zone A more accurately represents the dorsal-caudal portion of the field in the NTS and zone E represents the more ventralrostral portion of the terminal field in the NTS. Since the NTS shape changes throughout its dorsal to ventral extent, we chose to combine two $50 \mu \mathrm{m}$ sections into each zone, thereby permitting analyses of labeled density across closely aligned regions of interest.

Zone $\mathrm{A}$ is characterized by the sections where the fourth ventricle occupies the largest medial-lateral extent, where the solitary tract extends from the rostral pole to the caudal NTS, where the spinal trigeminal tract extends to near the rostral pole of the NTS, and by a lack of the hypoglossal and facial nuclei (Fig. $3 A$ ). Zone B also has the fourth ventricle extending in the medial-lateral plane, but less so than in zone A, the solitary tract is confined to the caudal portion of the NTS, the NTS extends to near the dorsal cochlear nucleus, and the spinal trigeminal tract extends just rostral to the rostral pole of the NTS. As in zone A, there is no hypoglossal nucleus; however, the dorsal-most extent of the facial nucleus may be present (Fig. 3B). Zone $\mathrm{C}$ is characterized by a significant thinning of the fourth ventricle compared with zones $\mathrm{A}$ and $\mathrm{B}$, the solitary tract extends throughout the NTS and is more medially located than in zones A and B, the rostral pole of the NTS extends to the level of dorsal cochlear nucleus, and both the hypoglossal and facial nuclei are evident. The spinal trigeminal tract extends beyond the rostral pole of the NTS in this zone (Fig. $3 C)$. Zone D is at the level ventral to the fourth ventricle, the NTS has elongated to the mid-region of the ventral cochlear nucleus and has narrowed significantly compared with the more dorsal zones. The hypoglossal and facial nuclei are clearly seen in this zone (Fig. 3D). Finally, in zone $\mathrm{E}$ the borders of the NTS are difficult to distinguish because of the many tracts and neuropil. However, compared with zone D, the NTS in zone E extends rostrally to the mid-rostral-caudal level of the ventral cochlear nucleus. The spinal trigeminal nucleus is less obvious in this zone compared with zone $\mathrm{D}$, and the hypoglossal and facial nuclei are larger and more distinct in this zone compared with the more dorsal zones (Fig. $3 E$ ). See Figure $3 F$ for relative positions of the zones within the NTS.

These landmarks were consistent among sham and nerve-cut groups. To check for reliability, a person naive to the groups assigned sections for each animal into the five zones, based on the characterization detailed above, and these were compared with the assignments made by the authors. With minor exceptions, the dorsal-ventral zone assignments among investigators were consistent.

Examination of terminal fields in coronal sections. The NTS from two control and two experimental animals were sectioned coronally on a vibratome at $50 \mu \mathrm{m}$ and imaged as described above. Coronal sections were used to examine the extent of chorda tympani nerve terminal field expansion with respect to the subdivisions of the NTS. No quantitative measurements were taken. Coronal sections were then stained for myelin (Schmued, 1990) following confocal microscopy to allow visualization of 

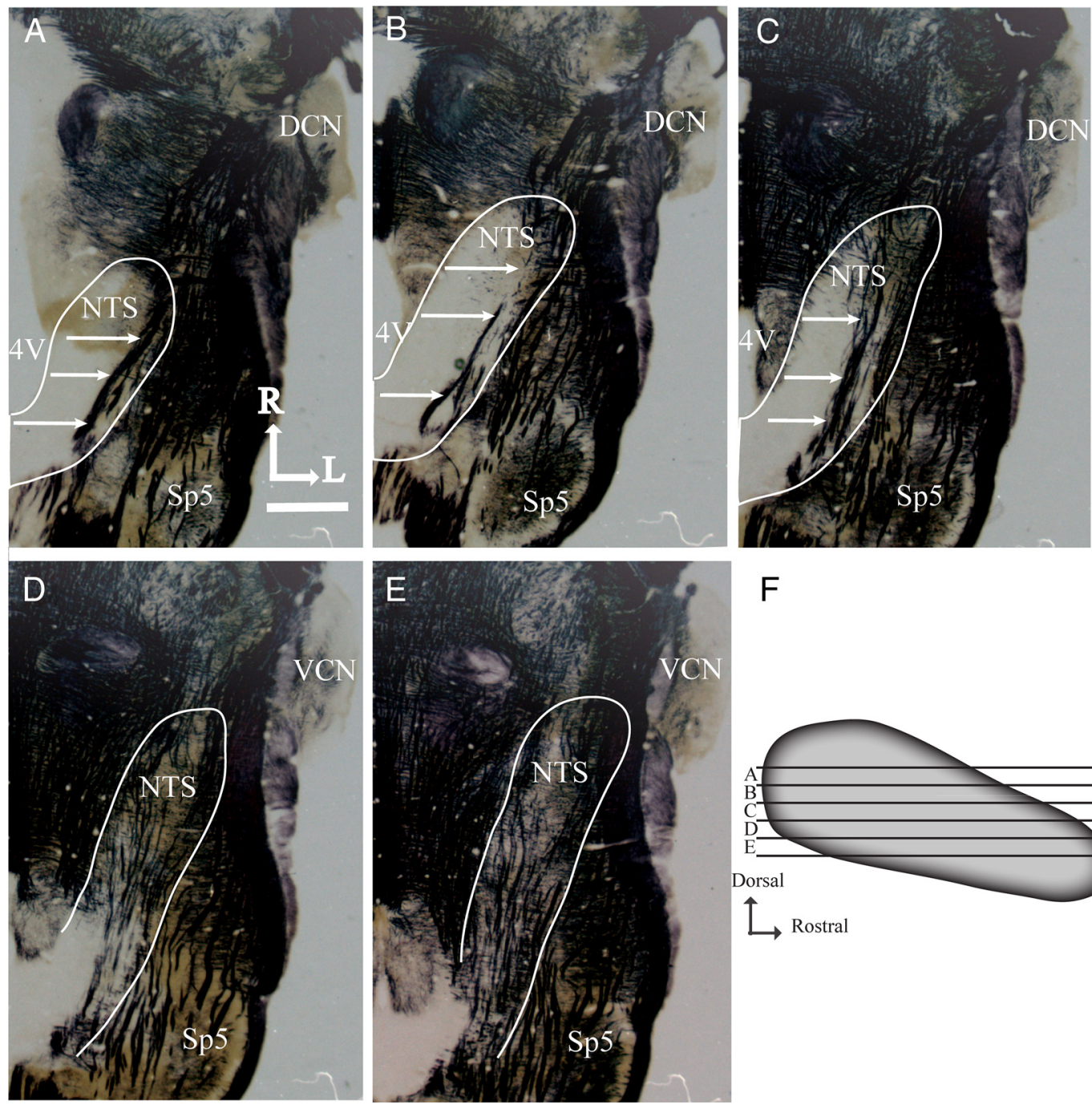

$\mathrm{F}$

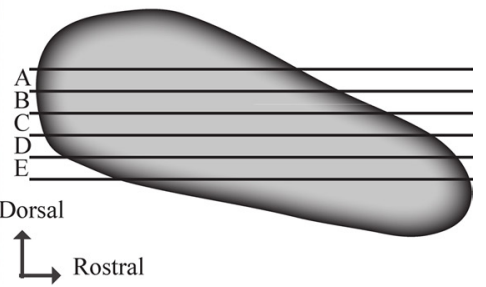

Figure 3. Myelin-stained horizontal brainstem sections and sagittal view of the nucleus of the solitary tract showing zones used for analysis. $\boldsymbol{A}-\boldsymbol{E}$ illustrates the shape of the NTS and other brainstem structures at five successive dorsal-ventral levels of NTS. The NTS is outlined in white, and white arrows point to the solitary tract. 4V, Fourth ventricle; Sp5, spinal trigeminal nucleus; DCN, dorsal cochlear nucleus; $V C N$, ventral cochlear nucleus; $\mathrm{R}$, rostral; L, lateral. Scale bar: (in $\boldsymbol{A}) \boldsymbol{A}-\boldsymbol{E}, 1 \mathrm{~mm}$. $\boldsymbol{F}$ shows the five dorsal-ventral levels of the NTS used for volume and density analysis with respect to the shape of the NTS. Each zone $(\boldsymbol{A}-\boldsymbol{E})$ represents $100 \mu \mathrm{m}$ of NTS.

labeled chorda tympani nerve terminal fields in anatomical subdivisions of NTS.

\section{Statistical analysis}

Terminal field volumes. The mean ( \pm SEM) was calculated for the total chorda tympani nerve terminal field volumes for all sections and for terminal field volumes within defined dorsal-ventral zones. Comparisons were made between experimental groups and sham controls using independent samples $t$ tests. Results from the post hoc analysis with $p<$ 0.05 are reported as significant.

Density by dorsal-ventral zones. Density measures were analyzed by zone within the $6 \times 7$ (column $\times$ row) grid by multivariate ANOVA (MANOVA) (SPSS; IBM Corporation). For the density analysis, we chose to show the partial $\eta$ squared value for each grid box to illustrate the location of the weightings that contribute to the overall statistical effect.

Assessment of regeneration of greater superficial petrosal and glossopharyngeal nerves and associated taste buds

The primary goal of this study was to examine the terminal field plasticity of the chorda tympani terminal field before the greater superficial petrosal and glossopharyngeal nerves functionally regenerated. Gustatory nerves and their associated taste buds undergo degeneration and then regeneration following nerve transection (Guth, 1957; Iwayama, 1970;
Cheal and Oakley, 1977; Cheal et al., 1977; St John et al., 2003). As an indicator of the degree of regeneration of the greater superficial petrosal and glossopharyngeal nerves, we used a combination of histological and neurophysiological measures. For the glossopharyngeal nerve, we examined the number and morphology of taste buds in foliate papillae ipsilateral to the nerve section (Fig. 1) in addition to recording neurophysiological taste responses from the sectioned glossopharyngeal nerve on and $\sim 35 \mathrm{~d}$ after surgery. To assess regeneration of the greater superficial petrosal nerve, we examined the number and morphology of taste buds in the nasoincisor duct (Fig. 1). Neurophysiological responses were not recorded from the greater superficial petrosal nerve.

Taste bud immunohistochemistry. We used anti-cytokeratin 19 and anticytokeratin 20, markers of intermediate filament proteins (Steinert et al., 1984), to stain for taste buds. Previous work showed the specificity of the immunoreactivity in rat lingual epithelium (Oakley et al., 1993; Zhang and Oakley, 1996; McCluskey and Hill, 2002; Hendricks et al., 2004).

The right greater superficial petrosal and glossopharyngeal nerves were sectioned, as described earlier, in animals at P15 $(n=6), \mathrm{P} 25(n=$ $4)$, or adulthood $(n=5)$. Thirty-five days later, animals were deeply anesthetized, perfused, and fixed with an acid-alcohol fixative. Tissue was obtained from the nasoincisor duct and foliate papillae and was sectioned at $10 \mu \mathrm{m}$ on a cryostat (Leica CM3050S) following sucrose infiltration (Barthel and Raymond, 1990). Tissue was incubated for $1 \mathrm{~h}$ in 
primary antibody (monoclonal anti-cytokeratin 4.62, 1:400, Sigma-Aldrich; monoclonal mouse anti-human cytokeratin 20, 1:25, Dako), followed by a $45 \mathrm{~min}$ incubation in secondary antibody (anti-mouse rhodamine-red, 1:500, Jackson ImmunoResearch), and was coverslipped using ProLong Gold (Invitrogen) before imaging on a fluorescent microscope.

All sections containing foliate papillae ipsilateral to the nerve cuts were imaged and analyzed for the presence of taste buds along the epithelium of the papilla. All sections containing the nasoincisor duct were imaged and examined for the presence of taste buds along the apical and ductal epithelia (El-Sharaby et al., 2004).

Whole nerve recordings from the glossopharyngeal nerve. Animals were deeply anesthetized with urethane $(1.6 \mathrm{mg} / \mathrm{kg})$, placed on a watercirculating heating pad to maintain body temperature and tracheotomized. The hypoglossal nerves were cut bilaterally to prevent tongue movements. To enhance access to the foliate papillae, sutures were placed around the lower jaw, in the anterior tongue, just rostral to the ipsilateral foliate papillae, and through the right cheek (Halsell et al., 1993; Travers and Norgren, 1995; Halsell and Travers, 1997), and were retracted to allow clear access to foliate papillae and to permit effective stimulation of the foliate taste buds. Following the ventral approach described previously for the glossopharyngeal nerve cut, the nerve was exposed, cut, and desheathed. The desheathed glossopharyngeal nerve was then placed on a platinum electrode with a reference electrode positioned in nearby muscle tissue. A mixture of petroleum jelly and mineral oil was placed in the cavity around the nerve to prevent drying.

Chemical grade reagents were used as taste stimuli and were dissolved in artificial saliva except for quinine hydrochloride, which was dissolved in distilled water. Responses to $2 \mathrm{M} \mathrm{NH}_{4} \mathrm{Cl}, 2 \mathrm{M} \mathrm{NaCl}, 2 \mathrm{M} \mathrm{KCl}$, and $0.1 \mathrm{M}$ quinine hydrochloride were recorded and analyzed with LabChart 6 (AD Instruments). Solutions were applied to the posterior tongue via syringe in $2 \mathrm{ml}$ aliquots and were followed by rinses with artificial saliva (Hellekant et al., 1985). Tactile responses induced by brushing a pipette along the foliate papillae were also recorded. For the purposes of this experiment, responses were quantified as present or absent to aid in determining the time course of glossopharyngeal nerve regeneration. Since our goal was to assess whether any functional regeneration occurred in the nerve, we chose to use relatively high stimulus concentrations and did not analyze relative response magnitudes. That is, we sought to answer the question, does the nerve respond to taste stimuli; not how does it respond?

Whole nerve recordings from the chorda tympani nerve. To examine potential nerve cut-induced changes in chorda tympani nerve function, we recorded whole nerve taste responses from the chorda tympani nerve in 22 additional rats at $\sim 35 \mathrm{~d}$ after nerve cut (P15 cut, $n=5$; P25 cut, $n=$ 3; adult cut, $n=5$; P15 sham, $n=2$; P25 sham, $n=3$; adult sham, $n=4$ ). Rats were prepared for recordings similar to that described for the glossopharyngeal nerve, with the exception that access to the chorda tympani nerve was gained by using a mandibular approach. The nerve was exposed near the tympanic bulla, cut, desheathed, and positioned on a platinum electrode. A second electrode was placed in nearby muscle to serve as ground. A mixture of petroleum jelly and mineral oil was placed in the cavity around the nerve.

All chemical stimuli were reagent grade and prepared in artificial saliva. Neural responses to a concentration series of $0.05,0.1,0.25$, and 0.5 $\mathrm{M} \mathrm{NaCl}$, sodium acetate, and $\mathrm{NH}_{4} \mathrm{Cl}$ were recorded. Each concentration series was bracketed by application of $0.5 \mathrm{M} \mathrm{NH}_{4} \mathrm{Cl}$. In addition, responses were recorded to $0.01 \mathrm{~N} \mathrm{HCl}$ and $1.0 \mathrm{M}$ sucrose. Solutions were applied to the tongue in $5 \mathrm{ml}$ aliquots with a syringe and allowed to remain on the tongue for $\sim 40 \mathrm{~s}$. After each solution application, the tongue was rinsed with artificial saliva for $\geq 1 \mathrm{~min}$. Chorda tympani nerve responses were calculated as follows: the mean height of the tonic response was measured from 5 to $15 \mathrm{~s}$ after stimulus application, and response magnitudes were expressed as ratios relative to the mean of the $0.5 \mathrm{M} \mathrm{NH}_{4} \mathrm{Cl}$ responses before and after stimulation. Response data were retained for analysis only when $0.5 \mathrm{M} \mathrm{NH}_{4} \mathrm{Cl}$ responses that bracketed a concentration series varied by $<10 \%$.

Statistical analysis. Neurophysiological responses were compared between nerve-sectioned and sham rats using independent-samples $t$ tests.
The $\alpha$ level was set at $p<0.05$ and was adjusted to compensate for the number of statistical tests conducted within a concentration series.

\section{Results}

Taste bud immunohistochemistry

Taste buds in sham rats were counted for each age group. There were no significant differences in the mean $( \pm$ SEM) number of taste buds among groups [nasoincisor duct (NID): P15, $46.5 \pm$ 12.5; P25, $32.5 \pm 6.5$; adult, $37.3 \pm 2.0$; Foliate: P15, $79.6 \pm 7.6$; $\mathrm{P} 25,98.3 \pm 13.3$, adult, $101.0 \pm 8.1]$. Therefore, all sham age group results were combined for this portion of data analysis (NID mean \pm SEM: $37.2 \pm 3.9, n=9$; Foliate mean \pm SEM: $92.6 \pm 5.7, n=14)$.

\section{Rats with nerve sections at P15}

Previous work in the gustatory system shows that the age of nerve section (chorda tympani or glossopharyngeal) plays a role in determining how many taste buds return when a cut nerve regenerates, with nerve cut at younger ages leading to less taste bud regeneration than following nerve cut at adulthood (Hosley et al., 1987; Sollars, 2005). Therefore, we expect fewer taste buds to regenerate in this group compared with the groups where nerves are cut in older animals.

Thirty-five days following greater superficial petrosal and glossopharyngeal nerve section at P15, the number of taste buds in the nasoincisor duct and in foliate papillae ipsilateral to the nerve cuts was reduced by $40 \%(p<0.05)$ and $75 \%(p<$ 0.0001 ), respectively, compared with sham rats. Qualitatively, the epithelial anatomy did not appear different from that in sham animals. For this study, rats underwent unilateral nerve sections; however, the nasoincisor duct is a bilaterally innervated structure (Miller and Spangler, 1982). Therefore, we expected $\sim 50 \%$ of taste buds to remain after unilateral nerve section since they were supported by the uncut nerve. This is in line with our results showing a $40 \%$ reduction in the number of taste buds in the nasoincisor duct, confirming that the greater superficial petrosal nerve that was sectioned had not regenerated at the time of assessment. Additionally, taste buds that remained present on the nasoincisor duct were restricted to the side contralateral to the nerve sections. The foliate taste buds that remained ipsilateral to the nerve section were not oriented correctly along the taste epithelium to allow access to taste stimuli. They were misshapen and taste pores were not readily identifiable, suggesting that they are unable to transmit incoming sensory information onto the brain. Taste pores were not counted, as the abnormal morphology of taste buds and lack of nerve function (see below) were strong indicators that regeneration of the glossopharyngeal nerve had not fully occurred.

\section{Rats with nerve sections at $P 25$}

Similarly, $35 \mathrm{~d}$ after greater superficial petrosal and glossopharyngeal nerve cut at $\mathrm{P} 25$ there was a significant reduction in the number of nasoincisor duct $(p<0.005)$ and foliate $(p<0.005)$ taste buds, representing a 63 and a $50 \%$ reduction compared with sham animals, respectively. Qualitatively and quantitatively, the remaining nasoincisor duct and foliate taste buds were similar to those seen in rats with nerve sections at P15.

\section{Rats with nerve sections at adulthood}

Finally, at $35 \mathrm{~d}$ after nerve section at adulthood, there was a significant reduction in the number of nasoincisor duct taste buds $(54 \%, p<0.05)$. However, there was not a significant difference in the number of foliate taste buds compared with shams $(10 \%$ increase, $p>0.05$ ). Qualitatively, these taste buds appeared dif- 


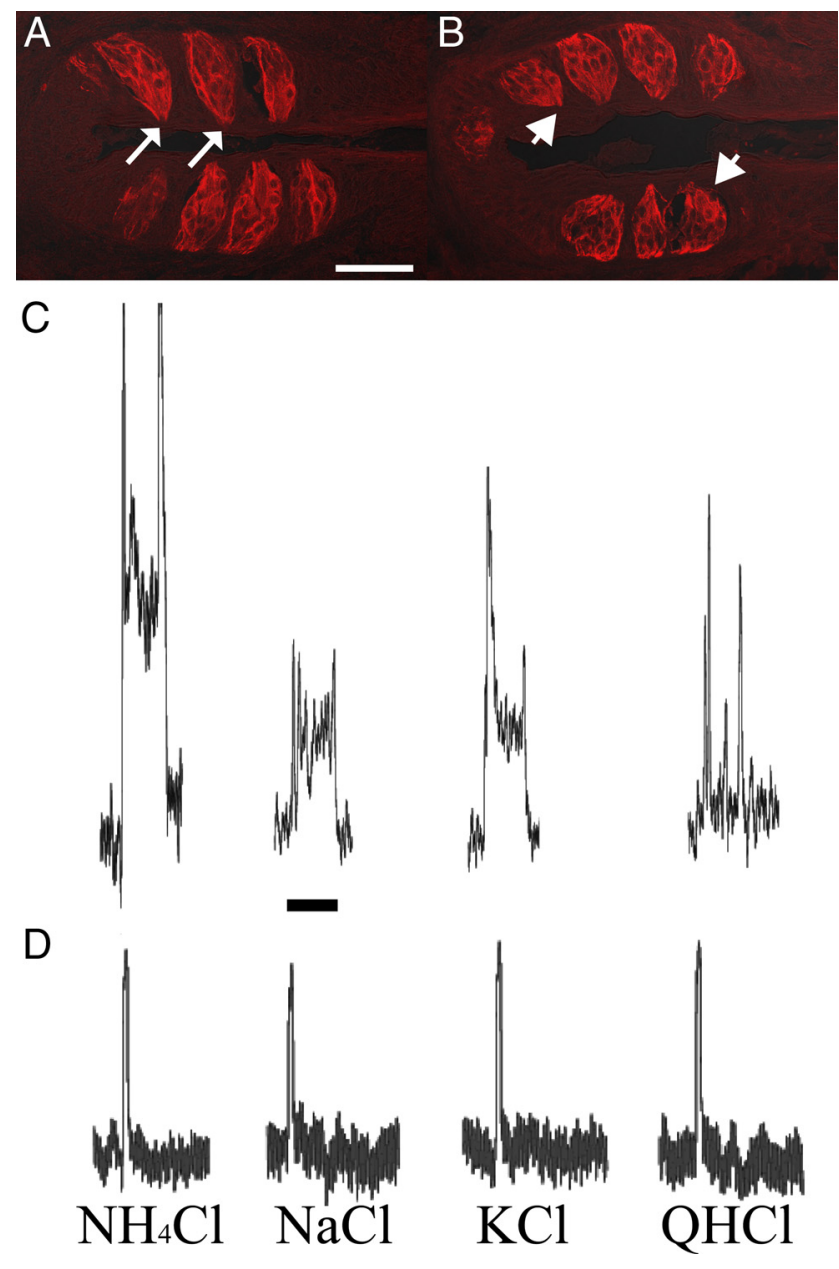

Figure 4. The glossopharyngeal nerve does not functionally regenerate within $35 \mathrm{~d}$ following nerve section. $\boldsymbol{A}$, Foliate taste buds from sham rats stained with cytokeratin-20 have a characteristic elongated shape and are oriented with taste pores (white arrows) adjacent to the epithelium. $\boldsymbol{B}$, In contrast, foliate taste buds from rats that underwent glossopharyngeal nerve section $35 \mathrm{~d}$ before tissue collection had taste buds with rounded shape, did not exhibit visible taste pores (white arrowheads) and were located further away from the epithelium compared with shams. $C$, Whole nerve recordings from the glossopharyngeal nerve of a sham rat showed robust, sustained taste responses to a variety of stimuli. $\boldsymbol{D}$, Whole nerve recordings from the glossopharyngeal nerve of a rat that underwent glossopharyngeal nerve section $35 \mathrm{~d}$ before recording showed no taste responses to a variety of taste stimuli. The transient responses to each stimulus in $\boldsymbol{C}$ and $\boldsymbol{D}$ likely reflect temperature and tactile responses. Scale bar: $A, 50 \mu \mathrm{m}$. Calibration: C, 15 s.

ferent from shams in that they were not oriented along the taste epithelium, their shape was rounded not elongated, and taste pores were not as readily identifiable as in sham rats (Fig. 4).

Since the number of foliate taste buds in adult nerve-cut animals was consistent with those of shams, we recorded whole nerve responses from the glossopharyngeal nerve to confirm that even though taste buds associated with the glossopharyngeal nerve were present, the nerve was not functional at $35 \mathrm{~d}$ after nerve cut.

\section{Whole nerve recordings from the glossopharyngeal nerve}

Recordings of glossopharyngeal nerve activity were obtained at $30 \mathrm{~d}(n=1), 35 \mathrm{~d}(n=3)$, and $42 \mathrm{~d}(n=1)$ after nerve cut in rats with nerve sections at adulthood to confirm that the nerve had not functionally regenerated at $35 \mathrm{~d}$ after the cut. Taste responses were not observed at any period examined at or before $35 \mathrm{~d}$ after glossopharyngeal nerve section, indicating that the nerve was not

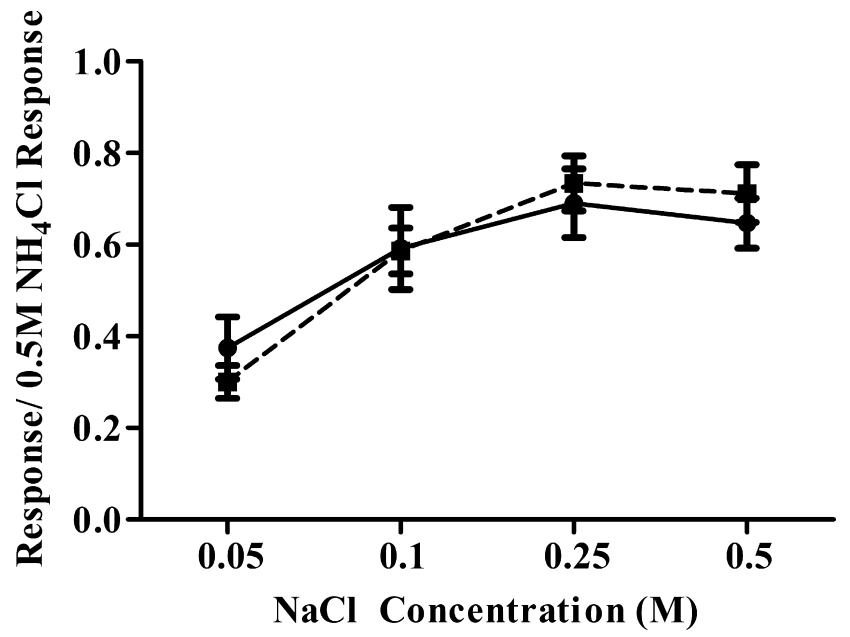

Figure 5. The function of the chorda tympani nerve is unaltered following greater superficial petrosal and glossopharyngeal nerve section. Whole nerve taste responses of the chorda tympani nerve to a concentration series of $\mathrm{NaCl}$ were unaffected by greater superficial petrosal and glossopharyngeal nerve cuts. In both control and nerve-cut animals, responses to $\mathrm{NaCl}$ increased to 0.25 m then plateaued. Closed circles, Control; closed squares, cut.

functional. Thus, even though taste buds were present at $35 \mathrm{~d}$ after nerve cut, taste information was not transmitted to the NTS. Figure $4 C$ shows representative responses of the glossopharyngeal nerve to $\mathrm{NH}_{4} \mathrm{Cl}, \mathrm{NaCl}, \mathrm{KCl}$, and quinine hydrochloride in a control animal. Figure $4 D$ shows representative responses to the same stimuli in an animal that had the glossopharyngeal nerve sectioned $35 \mathrm{~d}$ before recording. There was no change in steadystate response from baseline following application of the stimulus, despite the presence of taste buds in the foliate papillae. At $42 \mathrm{~d}$ after nerve cut, we were able to record weak taste responses from the glossopharyngeal nerve, indicating that the nerve does maintain the ability to regenerate and that our terminal field labels were done before functional regeneration.

To summarize, at $35 \mathrm{~d}$ after nerve cut there is evidence for incomplete regeneration of the glossopharyngeal nerve (presence of taste buds in comparable numbers to control) but no evidence that the nerve is transmitting afferent taste information. We felt it was not necessary to do these experiments in the P15 cut and P25 cut groups because there was no recovery of taste buds. This set of experiments gave us confidence that the greater superficial petrosal and glossopharyngeal nerves had not regenerated at the time we examined the chorda tympani nerve terminal field.

\section{Whole nerve recordings from the chorda tympani nerve}

No differences in chorda tympani nerve response magnitudes to taste stimuli existed among the three experimental groups (i.e., $\mathrm{P} 15, \mathrm{P} 25$, adult cuts) and among the respective three sham control groups. Therefore, we pooled the data for each group for analysis.

Figure 5 shows mean $( \pm$ SEM) relative response magnitudes from the chorda tympani nerve to a concentration series of $\mathrm{NaCl}$ for sham and cut rats. For both groups, response magnitudes increased with increasing stimulus concentration up to $0.25 \mathrm{M}$ $\mathrm{NaCl}$. Increasing stimulus concentration beyond $0.25 \mathrm{M}$ did not produce increased response magnitudes in both cut and sham rats (Fig. 5). No significant differences existed between cut and sham rats for any stimulus, including those to $0.01 \mathrm{~N} \mathrm{HCl}$ and 1.0 M sucrose $(p>0.10)$. Although these recordings do not allow a comparison of absolute activity levels, they provide evidence that the chorda tympani nerve had normal function in nerve-cut rats. 


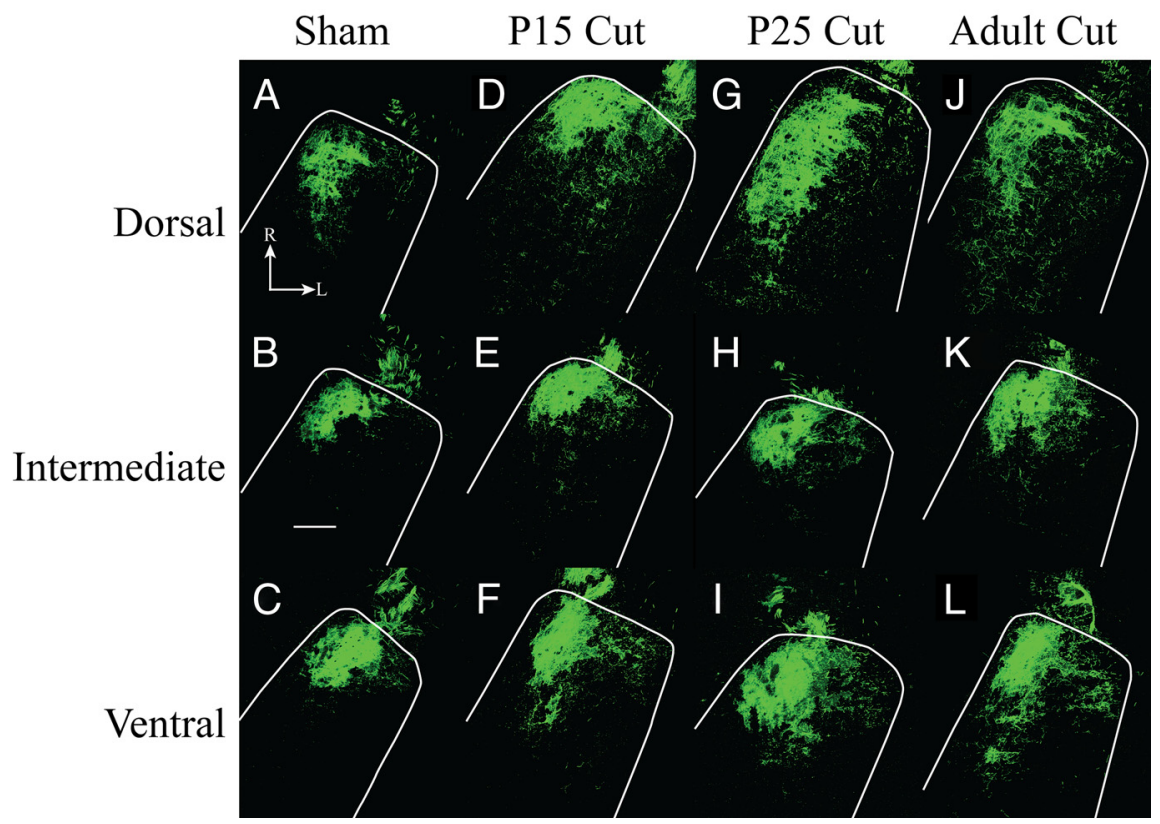

Figure 6. Horizontal sections of labeled chorda tympani nerve terminal field. $A-J$, Fluorescent micrographs are shown for sham $(\boldsymbol{A}-\boldsymbol{C}), \mathrm{P} 15$ cut $(\boldsymbol{D}-\boldsymbol{F}), \mathrm{P} 25$ cut $(\boldsymbol{G}-\boldsymbol{I})$, and adult cut $(\boldsymbol{J}-\boldsymbol{L})$ rats. The location of the NTS is outlined in white. $\boldsymbol{A}, \boldsymbol{D}, \mathbf{G}, \boldsymbol{J}$, The dorsal zone of labeled chorda tympani nerve terminal fields expanded in the caudal and lateral directions in all groups compared with sham. $\boldsymbol{B}$, $\boldsymbol{E}, \boldsymbol{H}, \boldsymbol{K}$, There was little expansion of the chorda tympani nerve terminal field in the intermediate zone. $\boldsymbol{C}, \boldsymbol{F}, \boldsymbol{I}, \boldsymbol{L}$, Ventral zone sections showed increased chorda tympani nerve terminal field across all groups compared with sham. Scale bar, $200 \mu \mathrm{m}$. R, Rostral; L, lateral.

\section{Chorda tympani nerve terminal field volume \\ Total terminal field}

Sham rats. There was no significant difference in terminal field volume between P15 and adult sham groups; therefore, terminal field results from sham groups were combined. All sham animals had chorda tympani nerve terminal field volumes comparable to adult controls from another study in our laboratory with the same imaging and analysis, but with different experimenters (our unpublished observation). This indicates no effect of sham surgery.

Rats with nerve sections at P15. Chorda tympani nerve terminal field volumes at adulthood in animals that had the greater superficial petrosal and glossopharyngeal nerves sectioned at P15 were significantly larger than shams $(p<0.05)$ (Fig. $6 A-F)$. Sham rat terminal fields represent just $26 \%$ of P15 cut terminal field volumes, indicating a large volumetric expansion of terminal field following nerve section (Fig. 7A) as well as a $34 \%$ increase in the number of physical sections $(50 \mu \mathrm{m})$ containing labeled terminal fields (Fig. 7B). Therefore, sectioning the greater superficial petrosal and the glossopharyngeal nerves at P15 resulted in a chorda tympani nerve terminal field volume approximately fourfold larger than would be expected if the animals were allowed to develop normally. These results infer that the normal developmental decrease in chorda tympani nerve terminal field volume was interrupted when the composite afferent input to the rostral NTS was altered at an early stage of terminal field maturation.

Rats with nerve sections at P25. The chorda tympani nerve terminal field volume of animals that had the greater superficial petrosal and glossopharyngeal nerves sectioned at P25 was significantly larger than shams $(p<0.0001)$ (Fig. 6A-C,G-I). Terminal field volume in sham rats was $27 \%$ of the terminal field volume in the P25 nerve section group, indicating that this group also had a chorda tympani nerve terminal field volume at adult- hood approximately four times larger than shams (Fig. 7A). The expansion of chorda tympani nerve terminal field in the P25 cut group mimicked the expansion seen in the P15 cut group. Again, there were a significantly greater number of physical sections that contained labeled terminal field compared with sham rats (32\% increase) (Fig. 7B). Combined, the results indicate that when the greater superficial petrosal and glossopharyngeal nerves were cut at P25, the uncut chorda tympani nerve terminal field not only expanded in volume, but also reorganized along the dorsal-ventral axis.

Rats with nerve sections at adulthood. Surprisingly, animals in which the greater superficial petrosal and the glossopharyngeal nerves were sectioned at adulthood also had chorda tympani nerve terminal field volumes significantly larger than sham controls $(p<0.05)$ (Fig. $6 A-C$, J$L$ ), indicating that the ability to respond to perturbations of the gustatory system was retained into adulthood. Sham control volumes represented $20 \%$ of the chorda tympani nerve terminal field volume in the adult cut group (Fig. $7 A$ ). As in the P15 and $\mathrm{P} 25$ cut groups, there was a significant increase in the number of physical sections containing terminal field label (32\% increase) (Fig. $7 B$ ). This again represents a spread of the chorda tympani nerve terminal field along the dorsal-ventral axis, as well as an increase in the volume of the terminal field, a pattern of expansion that followed results seen in the P15 and P25 nerve-cut groups.

Although this study was not designed to critically evaluate when the chorda tympani nerve terminal field changed after nerve section, we took a cursory look at rats with nerve sections at adulthood to examine the time course of field expansion. We examined total chorda tympani nerve terminal field volumes at $5 \mathrm{~d}(n=2), 6 \mathrm{~d}(n=3), 8 \mathrm{~d}(n=2), 10 \mathrm{~d}(n=3)$, and $15 \mathrm{~d}(n=$ 2) after nerve cut. We then determined the exponential line of best fit (plateau followed by one phase association) and found that the plateau $\left(4.7 \times 10^{6} \mu \mathrm{m}^{3}\right)$ that approximated the mean total chorda tympani nerve terminal field occurred at $\sim 15 \mathrm{~d}$ following nerve cut $\left(R^{2}=0.35\right)$ (Fig. 8). Therefore, based on this examination, changes in adult-sectioned induced expansion of the chorda tympani nerve terminal field occurred well before we took our measurements (at $35 \mathrm{~d}$ after nerve cut).

As an aside, we also took a cursory examination of the chorda tympani nerve terminal field in the NTS contralateral to the nerve sections in adult-sectioned rats $(n=3)$. For each rat, the total terminal field volume was similar to that found in sham rats, indicating that the effects noted here are confined to the chorda tympani nerve terminal field ipsilateral to the nerve sections.

\section{Spread of expanded terminal field in the horizontal plane}

To further examine the expanded terminal field in the horizontal plane, the density of label was calculated for sham and cut groups that survived $35 \mathrm{~d}$ after surgery. We did not analyze the very dorsal and very ventral regions of the NTS, as these areas contained labeled chorda tympani nerve terminal field in cut groups only. However, we reiterate that the finding of labels in these 

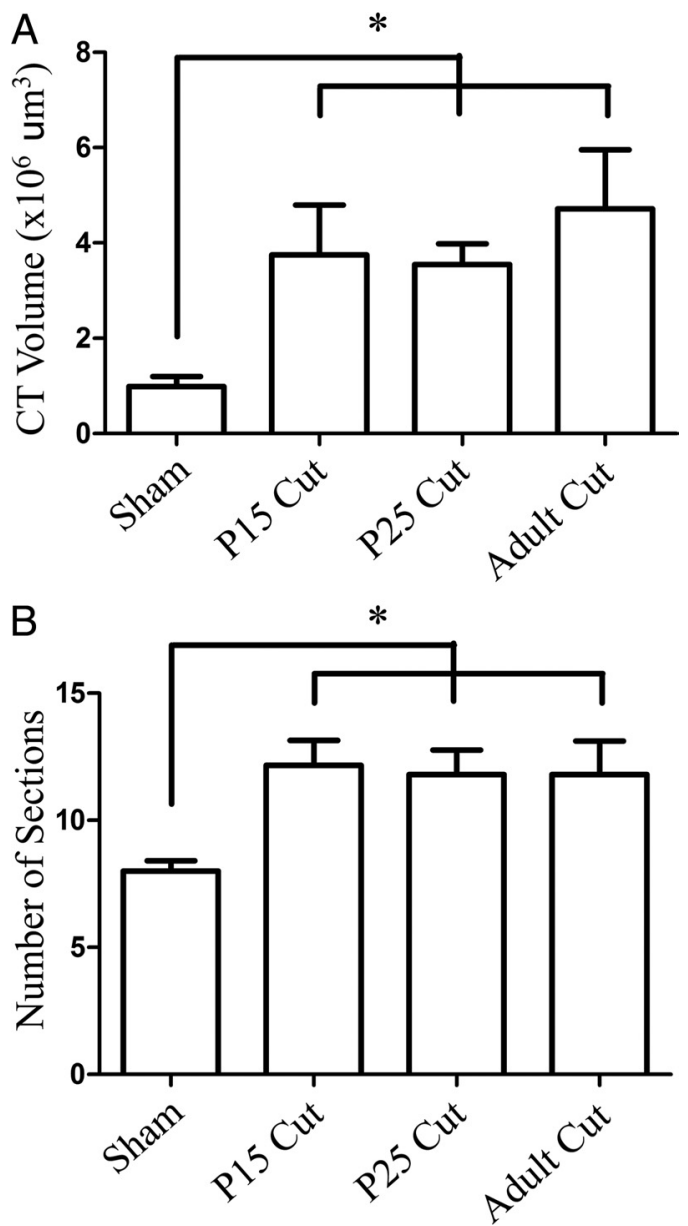

Figure 7. Mean chorda tympani nerve terminal field volumes and number of horizontal sections containing labeled terminal field. $A$, Total mean $( \pm S E M)$ terminal field volume was significantly increased in all nerve-cut groups compared with sham rats. $\boldsymbol{B}$, The mean ( \pm SEM) number of sections that contained labeled chorda tympani nerve terminal field was significantly increased in all nerve-cut groups. ${ }^{*} p<0.05$.

regions suggests a rearrangement of terminal field along the dorsal-ventral axis.

To illustrate the results of this analysis, we will present the results from sham rats and then from the adult nerve-cut group first, because the latter group underwent the greatest degree of chorda tympani nerve terminal field expansion.

Sham rats. In sham rats, the chorda tympani nerve terminal field label was found along the dorsal-ventral extent (Fig. 9F-J) of the NTS zones analyzed but was mainly restricted to the central three zones (zones B-D) (Fig. 9G-I). The densest areas of labeled chorda tympani nerve terminal field were found in these central regions, and labeling was concentrated in the rostral, medial portions of the NTS (also see Fig. 6). Little to no labeling was located in the caudal or lateral portions of the NTS.

Rats with nerve sections at adulthood. Rats that received nerve cut at adulthood had a significantly greater volume of labeled chorda tympani nerve terminal field in zone A compared with sham control rats $(6.8$-fold greater; $p<0.05)$ (Fig. 9A). In this zone, the terminal field was primarily found in the rostral, medial portion of the NTS similar to that seen in sham rats (Fig. $9 F, K$ ) but was much denser than the labeling found in the same region in sham animals. Significant differences in the density of the labeling in this zone were found also in the medial and caudal portions of the NTS (Fig. 9P). This analysis is consistent with the

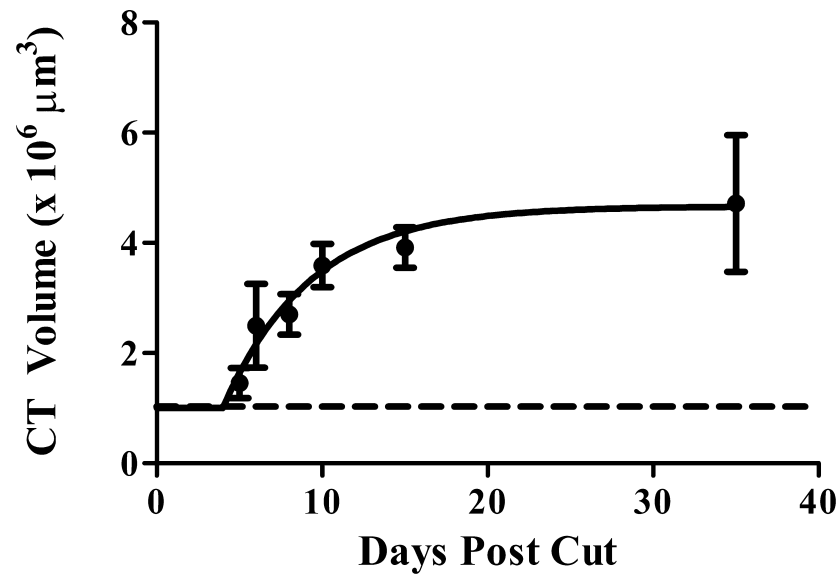

Figure 8. The chorda tympani nerve terminal field expands by $15 \mathrm{~d}$ after nerve cut. Total chorda tympani nerve terminal field volume was examined at 5, 6, 8, 10, and $15 \mathrm{~d}$ after nerve cut. An exponential line of best fit (plateau followed by one phase association) determined that the maximal expansion of chorda tympani nerve terminal field volume is complete within $15 \mathrm{~d}$ following greater superficial petrosal and glossopharyngeal nerve section. Closed circles, Adult cuts; dotted line, sham.

spread of the terminal field examined in the photomicrographs presented in Figure 6, $A$ and $J$. In zone B, a similar pattern to zone A occurred. There was a greater volume of labeled chorda tympani nerve terminal field in this zone for both groups (Fig. 9A,B), and, like in zone A, there was significantly more labeling (6.5fold) in the adult nerve-cut group compared with shams (Fig. $9 G, L)$, with the label densest in the rostral, medial portion of the NTS. As in zone A, significant increases in the density of labeling were also found in the more caudal portions of the NTS in the adult nerve-cut group compared with sham controls (Fig. 9Q). In zone $\mathrm{C}$, we found the greatest amount of terminal field labeling for both groups, but a significantly greater volume of labeled chorda tympani nerve terminal field in the adult nerve-cut group (2.6-fold) (Fig. 9C). At this level, the labeling was again mainly concentrated in the rostral, medial portions of the NTS (Fig. $9 M)$, but we did not find any significant changes in density at any locations compared with controls (Fig. 9R). In zone D, there was a significantly greater volume of labeled chorda tympani nerve terminal field in the cut group compared with sham controls (1.6-fold) (Fig. 9D). Again, the labeling was concentrated in the rostral, medial portion of the NTS in the nerve-cut group (Fig. $9 N)$, where the labeling was much denser than in the sham control group (Fig. 9S). At this more ventral zone of NTS, the spread of chorda tympani nerve terminal was not as great as the spread at the dorsal levels, which is consistent with the photomicrographs presented in Figure 6, $J-L$. In zone E, there was very little volume of terminal field in both sham and cut groups, and no difference was found between them (Fig. 9E). However, consistent with other levels of NTS, the labeling in the cut groups was concentrated in the rostral, medial portions of NTS (Fig. 90) and was denser than that of the shams in these regions (Fig. 9T).

In summary, the results shown here indicate that the adult chorda tympani nerve terminal field expanded primarily in the dorsal regions of NTS following greater superficial petrosal and glossopharyngeal nerve cut, although there were also ventrally located changes. Within these zones, there often was a spread of terminal field to more caudally located territory, areas unoccupied in sham controls. Interestingly, the zone with the largest amount of labeling (zone C) in both groups was the least affected by the experimental manipulation. It is noteworthy that in addi- 
Means
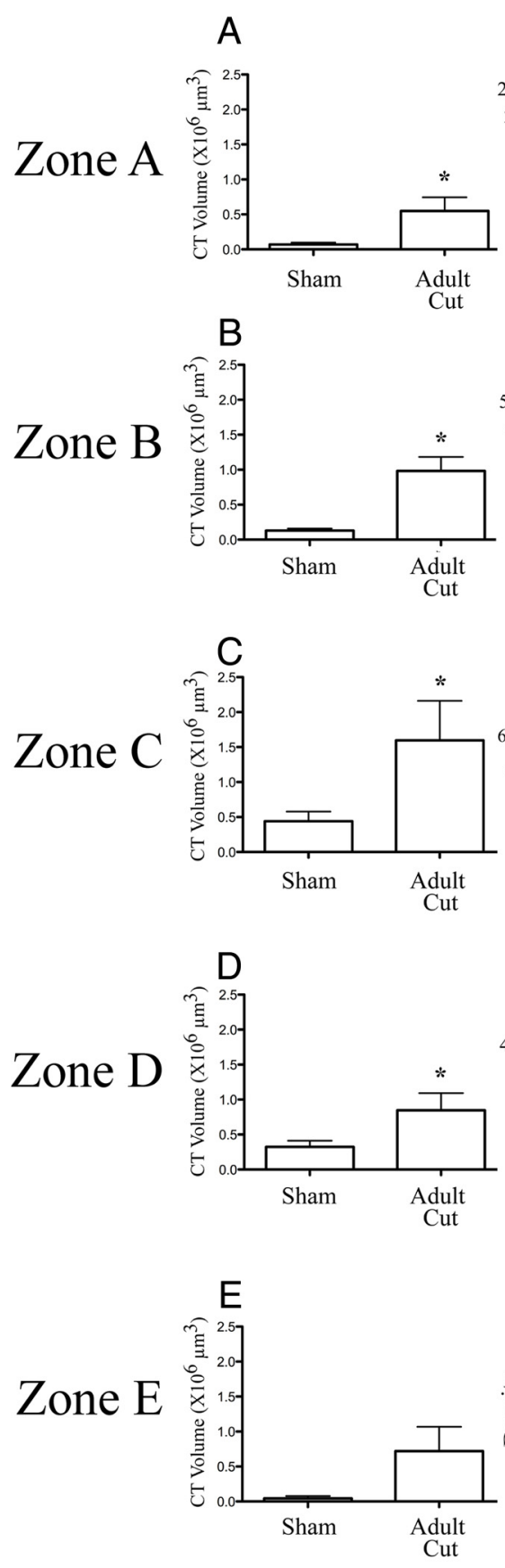

Sham
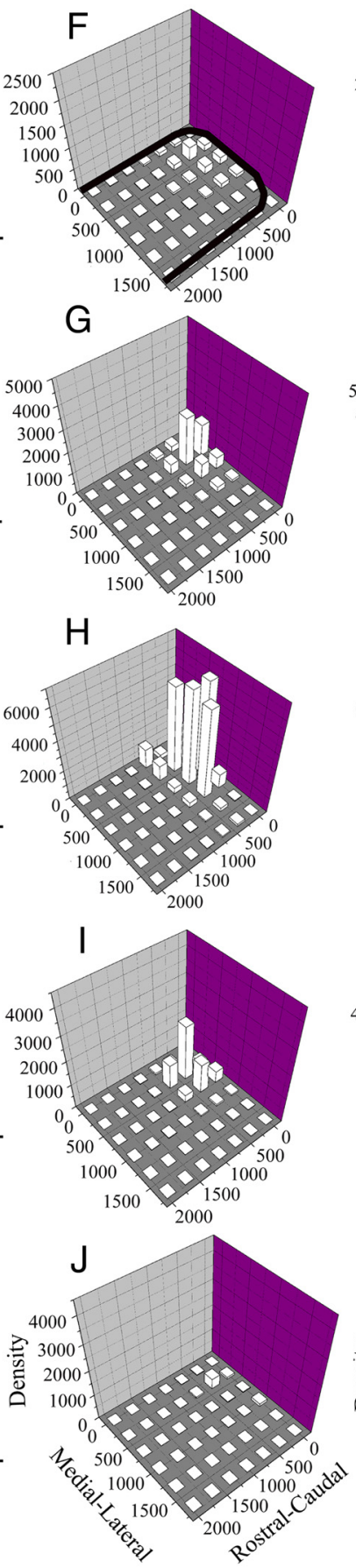

Adult Cut
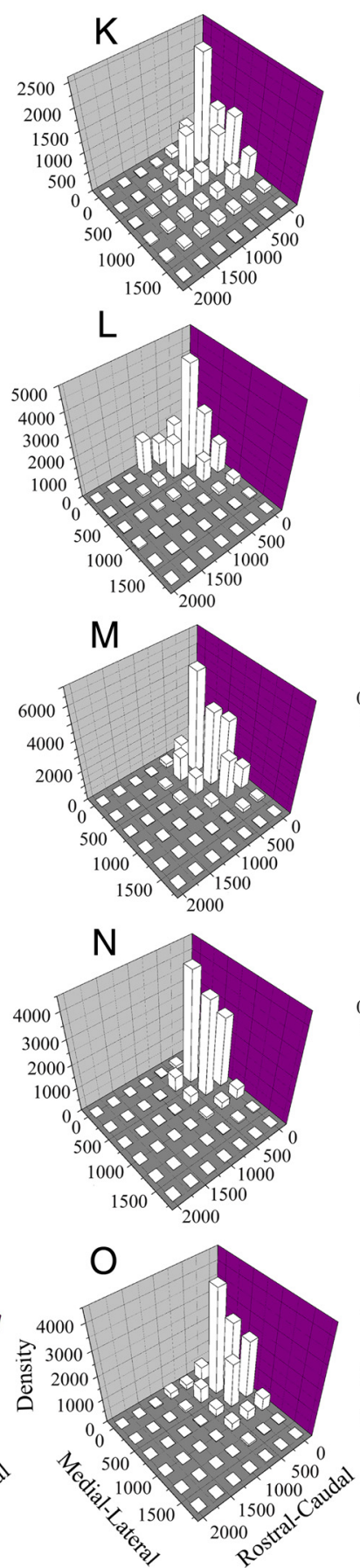

Partial eta ${ }^{2}$
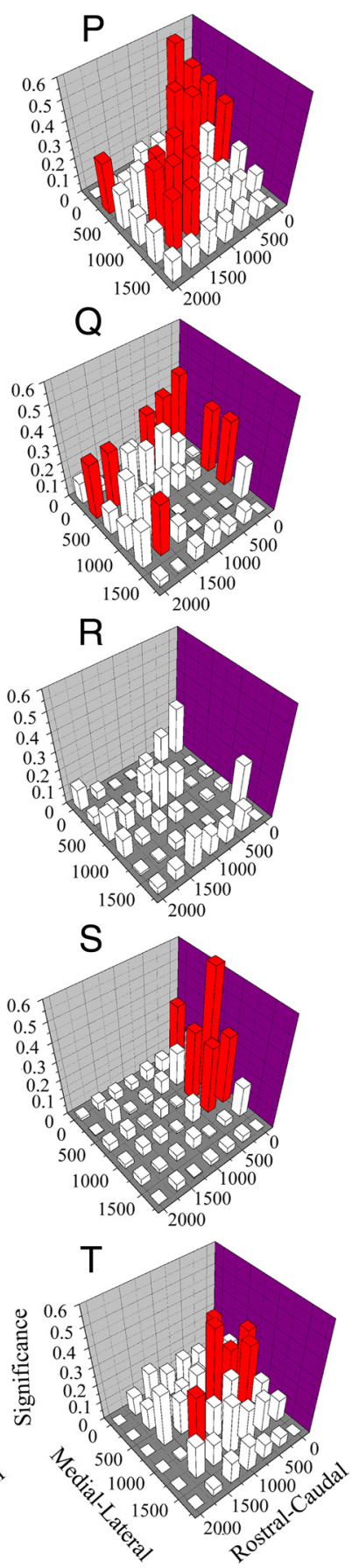

Figure 9. Chorda tympani nerve terminal field volumes and densities in $x, y$, and $z$ planes in adult cut rats compared with sham controls. $\boldsymbol{A}-\boldsymbol{E}$, Mean ( \pm SEM) chorda tympani nerve terminal field volumes in cut rats are greater than sham controls in four of five dorsal-ventral zones. $\boldsymbol{F}-\mathbf{0}$, Density of terminal field label is shown for sham $(\boldsymbol{F}-\boldsymbol{J})$ and adult cut rats $(\boldsymbol{K}-\mathbf{0})$. The medial-lateral axis represents distance $(\boldsymbol{\mu}$ m) from the medial border of the NTS and rostral-caudal axis represents distance $(\mu \mathrm{m})$ from the rostral pole of the NTS (see axes labels in $\boldsymbol{J}$ and $\boldsymbol{0}$ ). The graphs are oriented along the same axis as the NTS is in the brainstem (see solid black line in $\boldsymbol{F}$ for outline of the NTS; also see Figs. 3 and 6). The $z$-axis represents the mean density of label ("Density" in $\boldsymbol{J}$ and $\boldsymbol{O}$ represents the volume of terminal field label/volume of the NTS contained within the grid box $\left.\times 10^{5} \mu \mathrm{m}^{3}\right)$. See Materials and Methods for further detail. In each dorsal-ventral zone, the label was denser in the $x$ and $y$ planes for the adult cut rats $(\boldsymbol{F}, \boldsymbol{K}, \mathbf{G}, \boldsymbol{L}, \boldsymbol{H}, \boldsymbol{M}, \boldsymbol{I}, \boldsymbol{N}$, $\boldsymbol{J}, \mathbf{0}) . \boldsymbol{P}-\boldsymbol{T}$ shows the magnitude of the statistical effect resulting from comparisons within each grid box for each dorsal-ventral level. The medial-lateral and rostral-caudal axes are the same as described for $\boldsymbol{F}$ - $\mathbf{0}$. The $z$-axis in $\boldsymbol{P}-\boldsymbol{T}$ show the partial $\eta$ squared values obtained from the MANOVA analysis. The greater the partial $\eta$ squared value (represented as "Significance" in $\boldsymbol{T}$ ), the more significant the differences were between groups for the respective grid box. Red bars represent $p<0.05$. With the exception of zone C, there were significant differences between the two groups across the X, Y plane.

tion to the zones analyzed here, there was a significant amount of terminal field labeling in adult nerve-cut rats dorsal and ventral to the labeling seen in sham controls. There was an average of 100 and $10 \mu \mathrm{m}$ of tissue containing labeled chorda tympani nerve terminal field more dorsal and ventral, respectively, to that found in sham rats.

Rats with nerve sections at P15. Rats that received nerve cut at P15 did not have differences in chorda tympani nerve terminal 

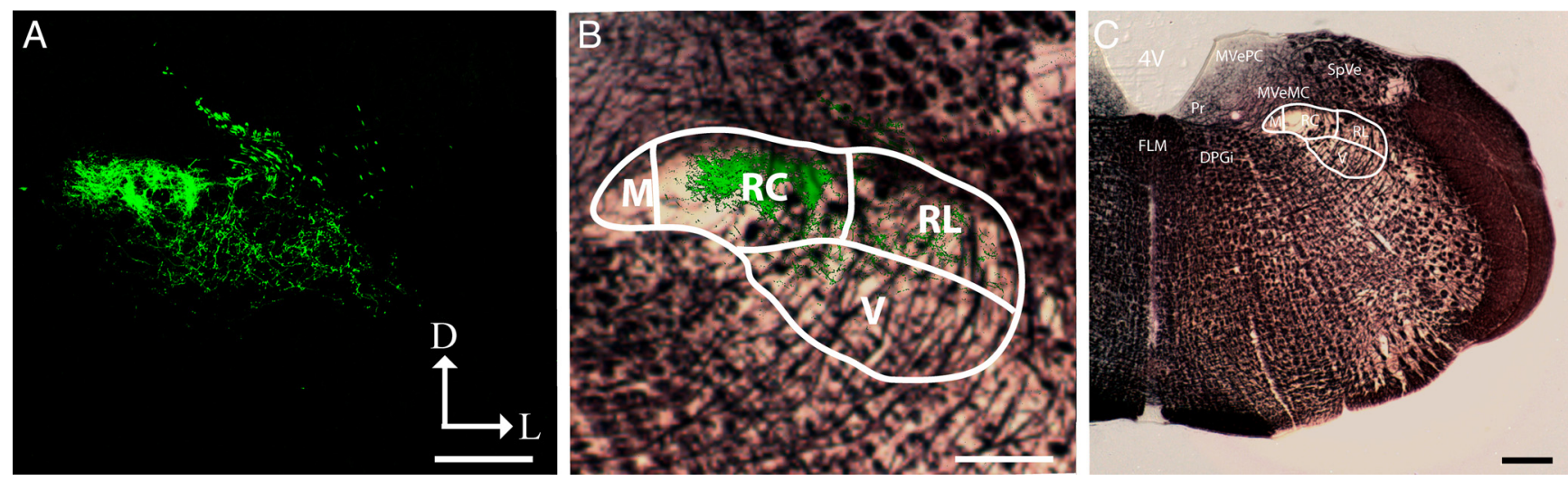

Figure 10. Chorda tympani nerve terminal field within NTS subdivisions: coronal sections. $\boldsymbol{A}$, The chorda tympani nerve terminal field of an adult cut rat was visualized in the coronal plane. $\boldsymbol{B}$, The terminal field was constrained to the rostral central and rostral lateral subdivisions. C, Myelin-stained sections show the location of the NTS relative to other brainstem structures. M, Medial subdivision of the NTS; RC, rostral central subdivision of the NTS; RL, rostral lateral subdivision of the NTS; V, ventral subdivision of the NTS; 4V, fourth ventricle; FLM, medial longitudinal fasciculus; DPGi, dorsal paragigantocellular nucleus; MVeMC, medial vestibular nucleus magnocellular; MVePC, medial vestibular nucleus parvocellular; $\mathrm{Pr}$, principal nucleus; $\mathrm{SpVe}, \mathrm{spinal}$ vestibular nucleus; $\mathrm{D}$, dorsal; L, lateral. Scale bars: $200 \mu \mathrm{m}$.

field volume in the most dorsal zones, zones A and B, but there were significant volumetric differences in zones C-E. In these three most ventral zones, the P15 cut group had significant increases in labeled densities throughout the rostral, medial, and caudal portions of the NTS. As in the adult cut group, the densest label was concentrated in the rostral, medial portion of the NTS. Moreover, in P15 cut rats, there was an average of $83 \mu \mathrm{m}$ of NTS that contained labeled chorda tympani nerve terminal field dorsal to zone A, and an average of $33 \mu \mathrm{m}$ of NTS containing labeling more ventral than zone E. The increase of label dorsal to zone A contributed greatly to both the increase in total volume and the increase in dorsal-ventral spread of terminal field.

Rats with nerve sections at P25. Rats that received nerve cuts at P25 had greater mean volumes of labeled chorda tympani nerve terminal field only in zones B and E compared with sham rats. However, significant increases in the density of the labeled terminal field were found across zones. Significant increases in density in zones A and B were found in the caudal portions of the NTS, while in zones D and $\mathrm{E}$ increases in density were found in the rostral, medial, and caudal regions of the NTS. The same pattern of labeling was seen in this group, where the densest label was concentrated in the rostral, medial portion of the NTS. Additionally, there were averages of 170 and $40 \mu \mathrm{m}$ of NTS containing labeled chorda tympani nerve terminal field more dorsally and ventrally, respectively, than found in sham rats.

\section{Terminal field expansion in the coronal plane}

To further examine the spread of expanded chorda tympani nerve terminal field following greater superficial petrosal and glossopharyngeal nerve sections, terminal field-labeled tissue from sham and adult nerve-cut rats was sectioned in the coronal plane.

Analyses showed that the chorda tympani nerve terminal field in sham control rats was restricted primarily to the rostrocentral and rostrolateral subdivisions of the NTS, consistent with an examination of chorda tympani nerve terminal field in coronal sections (Contreras et al., 1982; Whitehead, 1990). Coronal analysis of adult nerve-cut animals showed that the dramatically expanded chorda tympani nerve terminal field was also restricted to the rostrocentral and rostrolateral subdivisions of the NTS (Fig. 10). This finding indicates that there is no invasion into inappro- priate territory of the NTS that is not normally occupied by chorda tympani nerve terminal field.

\section{Comparison to immature chorda tympani nerve terminal field}

Since all three cut groups had similar chorda tympani nerve terminal fields, there may be a return to an immature state (i.e., large volume and spread) after the greater superficial petrosal and glossopharyngeal nerves are sectioned. That is, with the loss of competition, the chorda tympani nerve terminal field appears to revert to an immature stage. We compared the expanded chorda tympani nerve terminal field in P15 cut, P25 cut, and adult cut rats with the chorda tympani terminal field in P15 rats $(n=7)$. Volumetrically, there was no significant difference between the immature terminal field mean total volume to that from any nerve-cut group (P15 control: $4.14 \pm 0.8 \mu \mathrm{m}^{3}$; P15 cut: $3.75 \pm$ $1.1 \mu \mathrm{m}^{3}$; P25 cut: $3.55 \pm 0.4 \mu \mathrm{m}^{3}$; adult cut: $4.72 \pm 1.2 \mu \mathrm{m}^{3}$; $p>$ $0.05)$. There were also no differences in the dorsal-ventral spread of labeled chorda tympani nerve terminal field. We further compared the spread and density of chorda tympani nerve terminal field in the horizontal plane between P15 control and cut groups and found only minor differences. These results show that by cutting the greater superficial petrosal and glossopharyngeal nerves, the chorda tympani nerve reverts to an immature state where it occupies a much larger portion of the NTS than in adulthood. These results support the hypothesis that interactions among the three gustatory nerves drive the developmental decrease of terminal field.

\section{Results summary}

Altering gustatory input to the rostral NTS at any age by sectioning the glossopharyngeal and greater superficial petrosal nerves dramatically affects the terminal field morphology of the intact chorda tympani nerve terminal field. This effect occurs within the first 2 weeks after nerve cut, well before regeneration of the sectioned nerves. Rats that received nerve cut had greater volumes, dorsal-ventral spread, and horizontal spread of chorda tympani nerve terminal field than sham rats. The expanded terminal fields in cut groups mimics those found in P15 control rats. 


\section{Discussion}

The current study shows that sectioning the glossopharyngeal and greater superficial nerves, which normally have overlapping terminal fields with themselves and with the chorda tympani nerve, results in a dramatically expanded terminal field volume of the intact chorda tympani nerve when examined $35 \mathrm{~d}$ following nerve cuts. This rearrangement occurred regardless of the age of the animal when the nerves were cut. The chorda tympani nerve terminal field volume in all cut groups was approximately fourfold larger than that of sham animals. Although the terminal fields expanded in all planes compared with sham controls (rostral-caudal, medial-lateral, and dorsal-ventral planes), the enlarged terminal field did not cross the boundaries of the NTS to invade subnuclei that do not typically receive chorda tympani nerve input. Rather, the enlarged chorda tympani nerve terminal fields found in cut animals occupied space occupied by the terminal field at early stages of development (Mangold and Hill, 2007). Indeed, the expanded chorda tympani nerve terminal field was not different from the immature terminal field in measures of volume, dorsal-ventral extent of the labeling, and spread in the horizontal plane.

Our density analyses of chorda tympani nerve terminal field provide new insights into the distribution and amount of label within the rostral NTS for both sham rats and for rats with nerve cuts. For all groups and all dorsal-ventral zones, we found that the highest density of terminal field label occurred in the rostral, medial portion of the NTS. This dense rostral, medial region has been seen qualitatively in other studies (Lasiter and Kachele, 1990; King and Hill, 1991; Krimm and Hill, 1997; Pittman and Contreras, 2002); however, this is the first study to quantify the amount of chorda tympani nerve terminal field label. Through the density analyses we found that, with the exception of the dorsal-ventral zone that contained the highest amount of terminal field label in sham and in adult cut rats, the label density in the rostral, medial area of the dorsal-ventral NTS zones was dramatically increased in all nerve-cut groups compared with sham controls. This indicates that the caudal and lateral spread of the chorda tympani nerve terminal field did not occur at the expense of label in the dense rostral, medial region.

Perhaps the most surprising finding of this study was that the chorda tympani nerve terminal field volume and the spread of the field in all nerve-cut groups were greater than in sham controls. We hypothesized that loss of input to the NTS from the greater superficial petrosal and glossopharyngeal nerves would alter the developmental pruning of the chorda tympani nerve terminal field (Mangold and Hill, 2008) by "freezing" the field at the developmental stage when the nerves were cut. For example, we expected the P25 cut group to have a terminal field size and distribution similar at $35 \mathrm{~d}$ after cut (i.e., at adulthood) to that found in animals aged P25, and that the adult nerve cuts would have no effect on the terminal field because the chorda tympani field was fully pruned by that age.

Our data not only show that our original hypotheses are incorrect, they provide a snapshot into how plastic the chorda tympani nerve terminal field remains throughout development. Since the dorsal region is occupied by all three terminal fields at the time of glossopharyngeal and greater superficial nerve cuts at P15 (Mangold and Hill, 2008), the terminal field expands into the intermediate and ventral regions of the NTS, where less overlap among the three fields exists. At P25, the chorda tympani nerve terminal field has undergone some developmental pruning across the dorsal-ventral extent of the NTS (Mangold and Hill,
2008), but the amount of pruning is not complete. For the P25 cut group, the terminal field expands to occupy regions from which it has been pruned (i.e., along the dorsal-ventral axis and in the rostral, medial and caudal regions of the NTS). Finally, the adult cut group has territory in the NTS into which it can expand in all three planes. In short, these dynamic changes suggest that without competition from overlapping terminal fields, the chorda tympani nerve terminal field reverts to its immature, prepruned state.

Following glossopharyngeal nerve transection, performance on many taste-related behavioral tasks is unaffected despite the loss of a large population of taste buds (St John et al., 1994, 2003; Spector, 2005). Our anatomical results suggest a functional explanation for this disparity. The expanded chorda tympani nerve terminal field may functionally compensate for the lost input from taste buds to maintain normal behavioral responses.

We propose that mechanisms related to competition among elements in the three overlapping terminal fields in the NTS account for the changes seen here. Work in other sensory systems strongly indicates that competition among afferent neurons for neurotrophins, synaptic space, and other neurally mediated factors refine their synaptic inputs, often in an activity-dependent manner (Murphey and Lemere, 1984; Shatz, 1990; Chiaia et al., 1992; Jacquin et al., 1993; Penn and Shatz, 1999; Foeller and Feldman, 2004; Demas et al., 2006; Gosse et al., 2008). For example, increased sprouting of intact primary somatosensory axonal arbors occurs after denervation of neighboring inputs in immature and mature systems (Chiaia et al., 1993; Renehan et al., 1994); these changes do not appear to be activity dependent (Henderson et al., 1992; Jacquin et al., 1993). In contrast, work in the visual system showed that the age-related refinement of retinal ganglion cell arbors is dependent both on activity-dependent (Sretavan and Shatz, 1986; Karmarkar and Dan, 2006) and activity-independent mechanisms (Wilkinson, 2000; Gosse et al., 2008).

Although not tested directly here, the density results are consistent with outcomes that would be attributable to competition. For example, we found in all cut groups that the chorda tympani nerve terminal field expanded dorsally into areas normally occupied by the glossopharyngeal nerve terminal field and had a similar topography of labeling throughout the NTS as seen for the glossopharyngeal nerve (May and Hill, 2006; Mangold and Hill, 2007, 2008). Similarly, the loss of competition through sectioning the greater superficial petrosal nerve, which normally shares a dense, rostral, medial region of its terminal field with the chorda tympani nerve (Mangold and Hill, 2007), may have contributed to the increase in chorda tympani nerve terminal field density in this location. Thus, loss of one or more inputs at any age may shift the balance of competition to the intact chorda tympani nerve, resulting in increased axonal growth and/or branching. Given that the chorda tympani nerve appears to be more plastic during development and following early dietary manipulations than the other two nerves (Mangold and Hill, 2007, 2008), it may be that the terminal fields from the other nerves may not expand like that of the chorda tympani nerve in this experimental paradigm.

The rearrangements reported here may relate to activitydependent and activity-independent mechanisms. If a loss of competing inputs onto target neurons in the NTS leads to new synapses and axonal processes from the spared chorda tympani nerve, such an increase in functional synaptic inputs could in turn lead to the release or enhanced uptake of axonal growth factors such as neurotrophins. Cutting the other two nerves could also lead to a local immune response that may induce a permis- 
sive environment for axonal growth and/or changes in the afferent signal carried by the chorda tympani nerve.

The resultant expansion of the terminal fields seen here is consistent with other studies of central gustatory development and plasticity that may also involve some level of competition. When the afferent gustatory system is perturbed, the chorda tympani nerve terminal field reverts to or exceeds an immature size by adulthood, primarily through the expansion of terminal fields in the dorsal zones of the NTS (King and Hill, 1991; Krimm and Hill, 1997; May and Hill, 2006; Mangold and Hill, 2007, 2008; May et al., 2008). For these studies, early developmental dietary manipulations produced attenuated neural responses and/or changes in the early environment of the peripheral and CNS that resulted in expanded terminal fields at adulthood (Sollars et al., 2006; Thomas and Hill, 2008). In some cases, the expanded terminal fields only occurred at adulthood (Mangold and Hill, 2008).

Although we show similarities in chorda tympani nerve terminal field among all cut groups and that the fields are similar to P15 control rats, the impact on the overall circuitry of the gustatory system at the level of the NTS and centrally may not be the same. That is, it is not clear whether the local and descending components of the circuits show the same types of plastic changes among groups. Some of the other components of the circuitry may be differentially less affected than the chorda tympani nerve terminal field with age. However, it is likely that the experimentally induced changes in the chorda tympani nerve terminal field have an impact on the overall circuitry of the rostral NTS and the functional processing of orosensory signals. The functional changes may in turn have significant consequences on agerelated behavioral taste preferences and aversions.

\section{References}

Barthel LK, Raymond PA (1990) Improved method for obtaining 3-microns cryosections for immunocytochemistry. J Histochem Cytochem 38:1383-1388.

Cheal M, Oakley B (1977) Regeneration of fungiform taste buds: temporal and spatial characteristics. J Comp Neurol 172:609-626.

Cheal M, Dickey WP, Jones LB, Oakley B (1977) Taste fiber responses during reinnervation of fungiform papillae. J Comp Neurol 172:627-646.

Chiaia NL, Bennett-Clarke CA, Eck M, White FA, Crissman RS, Rhoades RW (1992) Evidence for prenatal competition among the central arbors of trigeminal primary afferent neurons. J Neurosci 12:62-76.

Chiaia NL, Bauer WR, Rhoades RW (1993) Prenatal development of the receptive fields of individual trigeminal ganglion cells in the rat. J Neurophysiol 69:1171-1180.

Contreras RJ, Beckstead RM, Norgren R (1982) The central projections of the trigeminal, facial, glossopharyngeal and vagus nerves: an autoradiographic study in the rat. J Auton Nerv Syst 6:303-322.

Demas J, Sagdullaev BT, Green E, Jaubert-Miazza L, McCall MA, Gregg RG, Wong RO, Guido W (2006) Failure to maintain eye-specific segregation in nob, a mutant with abnormally patterned retinal activity. Neuron 50:247-259.

El-Sharaby A, Ueda K, Wakisaka S (2004) Immunohistochemical distribution of growth-associated protein 43 (GAP-43) in developing rat nasoincisor papilla. Anat Rec A Discov Mol Cell Evol Biol 277:370-383.

Ferrell MF, Mistretta CM, Bradley RM (1981) Development of chorda tympani taste responses in rat. J Comp Neurol 198:37-44.

Foeller E, Feldman DE (2004) Synaptic basis for developmental plasticity in somatosensory cortex. Curr Opin Neurobiol 14:89-95.

Gosse NJ, Nevin LM, Baier H (2008) Retinotopic order in the absence of axon competition. Nature 452:892-895.

Guth L (1957) The effects of glossopharyngeal nerve transection on the circumvallate papilla of the rat. Anat Rec 128:715-731.

Halsell CB, Travers SP (1997) Anterior and posterior oral cavity responsive neurons are differentially distributed among parabrachial subnuclei in rat. J Neurophysiol 78:920-938.

Halsell CB, Travers JB, Travers SP (1993) Gustatory and tactile stimulation of the posterior tongue activate overlapping but distinctive regions within the nucleus of the solitary tract. Brain Res 632:161-173.

Hellekant G, af Segerstad CH, Roberts T, van der Wel H, Brouwer JN, Glaser D, Haynes R, Eichberg JW (1985) Effects of gymnemic acid on the chorda tympani proper nerve responses to sweet, sour, salty and bitter taste stimuli in the chimpanzee. Acta Physiol Scand 124:399-408.

Henderson TA, Woolsey TA, Jacquin MF (1992) Infraorbital nerve blockade from birth does not disrupt central trigeminal pattern formation in the rat. Brain Res Dev Brain Res 66:146-152.

Hendricks SJ, Brunjes PC, Hill DL (2004) Taste bud cell dynamics during normal and sodium-restricted development. J Comp Neurol 472:173-182.

Hill DL, Almli CR (1980) Ontogeny of chorda tympani nerve responses to gustatory stimuli in the rat. Brain Res 197:27-38.

Hosley MA, Hughes SE, Morton LL, Oakley B (1987) A sensitive period for the neural induction of taste buds. J Neurosci 7:2075-2080.

Iwayama T (1970) Changes in the cell population of taste buds during degeneration and regeneration of their sensory innervation. Z Zellforsch Mikrosk Anat 110:487-495.

Jacquin MF, Zahm DS, Henderson TA, Golden JP, Johnson EM, Renehan WE, Klein BG (1993) Structure-function relationships in rat brainstem subnucleus interpolaris. X. Mechanisms underlying enlarged spared whisker projections after infraorbital nerve injury at birth. J Neurosci 13:2946-2964.

Karmarkar UR, Dan Y (2006) Experience-dependent plasticity in adult visual cortex. Neuron 52:577-585.

King CT, Hill DL (1991) Dietary sodium chloride deprivation throughout development selectively influences the terminal field organization of gustatory afferent fibers projecting to the rat nucleus of the solitary tract. J Comp Neurol 303:159-169.

Krimm RF, Hill DL (1997) Early prenatal critical period for chorda tympani nerve terminal field development. J Comp Neurol 378:254-264.

Lasiter PS, Kachele DL (1990) Effects of early postnatal receptor damage on development of gustatory recipient zones within the nucleus of the solitary tract. Brain Res Dev Brain Res 55:57-71.

Mangold JE, Hill DL (2007) Extensive reorganization of primary afferent projections into the gustatory brainstem induced by feeding a sodiumrestricted diet during development: less is more. J Neurosci 27: $4650-4662$.

Mangold JE, Hill DL (2008) Postnatal reorganization of primary afferent terminal fields in the rat gustatory brainstem is determined by prenatal dietary history. J Comp Neurol 509:594-607.

May OL, Hill DL (2006) Gustatory terminal field organization and developmental plasticity in the nucleus of the solitary tract revealed through triple-fluorescence labeling. J Comp Neurol 497:658-669.

May OL, Erisir A, Hill DL (2008) Modifications of gustatory nerve synapses onto nucleus of the solitary tract neurons induced by dietary sodiumrestriction during development. J Comp Neurol 508:529-541.

McCluskey LP, Hill DL (2002) Sensitive periods for the effect of dietary sodium restriction on intact and denervated taste receptor cells. Am J Physiol Regul Integr Comp Physiol 283:R1275-R1284.

Miller IJ, Spangler KM (1982) Taste bud distribution and innervation on the palate of the rat. Chem Senses 7:99-108.

Murphey RK, Lemere CA (1984) Competition controls the growth of an identified axonal arborization. Science 224:1352-1355.

Oakley B, Lawton A, Riddle DR, Wu LH (1993) Morphometric and immunocytochemical assessment of fungiform taste buds after interruption of the chorda-lingual nerve. Microsc Res Tech 26:187-195.

Pallas SL, Hoy RR (1986) Regeneration of normal afferent input does not eliminate aberrant synaptic connections of an identified auditory interneuron in the cricket, Teleogryllus oceanicus. J Comp Neurol 248:348-359.

Penn AA, Shatz CJ (1999) Brain waves and brain wiring: the role of endogenous and sensory-driven neural activity in development. Pediatr Res 45:447-458.

Pittman DW, Contreras RJ (2002) Dietary NaCl influences the organization of chorda tympani neurons projecting to the nucleus of the solitary tract in rats. Chem Senses 27:333-341.

Renehan WE, Crissman RS, Jacquin MF (1994) Primary afferent plasticity following partial denervation of the trigeminal brainstem nuclear complex in the postnatal rat. J Neurosci 14:721-739. 
Sahoo PK, Soltani S, Wong KC, Chen YC (1988) A survey of thresholding techniques. Comput Vis Graphics Image Process 41:233-260.

Schmued LC (1990) A rapid, sensitive histochemical stain for myelin in frozen brain sections. J Histochem Cytochem 38:717-720.

Shatz CJ (1990) Competitive interactions between retinal ganglion cells during prenatal development. J Neurobiol 21:197-211.

Sollars SI (2005) Chorda tympani nerve transection at different developmental ages produces differential effects on taste bud volume and papillae morphology in the rat. J Neurobiol 64:310-320.

Sollars SI, Walker BR, Thaw AK, Hill DL (2006) Age-related decrease of the chorda tympani nerve terminal field in the nucleus of the solitary tract is prevented by dietary sodium restriction during development. Neuroscience 137:1229-1236.

Spector AC (2005) The functional consequences of gustatory nerve regeneration as assessed behaviorally in a rat model. Chem Senses 30 [Suppl 1]:i66-i67.

Sretavan DW, Shatz CJ (1986) Prenatal development of cat retinogeniculate axon arbors in the absence of binocular interactions. J Neurosci 6:9901003.

Steinert PM, Jones JC, Goldman RD (1984) Intermediate filaments. J Cell Biol 99:22s-27s

St John SJ, Garcea M, Spector AC (1994) Combined, but not single, gusta- tory nerve transection substantially alters taste-guided licking behavior to quinine in rats. Behav Neurosci 108:131-140.

St John SJ, Garcea M, Spector AC (2003) The time course of taste bud regeneration after glossopharyngeal or greater superficial petrosal nerve transection in rats. Chem Senses 28:33-43.

Thomas JE, Hill DL (2008) The effects of dietary protein restriction on chorda tympani nerve taste responses and terminal field organization. Neuroscience 157:329-339.

Travers SP, Norgren R (1995) Organization of orosensory responses in the nucleus of the solitary tract of rat. J Neurophysiol 73:2144-2162.

Whitehead MC (1990) Subdivisions and neuron types of the nucleus of the solitary tract that project to the parabrachial nucleus in the hamster. J Comp Neurol 301:554-574.

Whitehead MC, Frank ME (1983) Anatomy of the gustatory system in the hamster: central projections of the chorda tympani and the lingual nerve. J Comp Neurol 220:378-395.

Wilkinson DG (2000) Topographic mapping: organising by repulsion and competition? Curr Biol 10:R447-R451.

Zhang C, Oakley B (1996) The distribution and origin of keratin 20 -containing taste buds in rat and human. Differentiation 61:121127. 